\title{
Miranda
}

Revue pluridisciplinaire du monde anglophone /

Multidisciplinary peer-reviewed journal on the English-

speaking world

15 | 2017

Lolita at 60 / Staging American Bodies

\section{Publicités, magazines, et autres textes non littéraires dans Lolita: pour une autre poétique intertextuelle}

\section{Marie Bouchet}

\section{OpenEdition}

Journals

\section{Édition électronique}

URL : http://journals.openedition.org/miranda/11145

DOI : 10.4000/miranda. 11145

ISSN : 2108-6559

\section{Éditeur}

Université Toulouse - Jean Jaurès

\section{Référence électronique}

Marie Bouchet, «Publicités, magazines, et autres textes non littéraires dans Lolita : pour une autre poétique intertextuelle », Miranda [En ligne], 15 | 2017, mis en ligne le 06 octobre 2017, consulté le 16 février 2021. URL : http://journals.openedition.org/miranda/11145 ; DOI : https://doi.org/10.4000/ miranda. 11145

Ce document a été généré automatiquement le 16 février 2021.

\section{cc) (1)}

Miranda is licensed under a Creative Commons Attribution-NonCommercial-NoDerivatives 4.0

International License. 


\title{
Publicités, magazines, et autres textes non littéraires dans Lolita: pour une autre poétique intertextuelle
}

\author{
Marie Bouchet
}

Si les critiques n'ont pas encore terminé l'annotation de Lolita ${ }^{1}$, roman dont l'épaisseur intertextuelle fait que de nombreuses références aux littératures anglaise, américaine, française, russe, espagnole, italienne, latine, orientales, etc. sont régulièrement débusquées et font montre à la fois de l'immense érudition de Nabokov et de son goût pour les énigmes et les jeux de mots, ces mêmes critiques se sont moins penchés sur un aspect tout à fait unique de l'intertextualité de ce grand roman, à savoir les modes d'insertion, dans le texte littéraire, de langages et d'images qui ne sont en principe pas les matériaux premiers de la littérature, car ils sont issus de chansons, de magazines, de guides de voyage, de la publicité, ou encore d'instructions pour utiliser des produits ou occuper des lieux. Si la critique a perçu dans cette abondance de références à the American way of life de l'après-seconde guerre mondiale une satire sociétale ${ }^{2}$, elle s'est rarement attachée à étudier d'un point de vue littéraire comment ces référents sont tissés dans la trame textuelle. Notre étude entend donc renouveler le regard porté sur ces référents en les analysant avec les mêmes outils que les allusions intertextuelles à Shakespeare, Poe ou Proust, et, in fine, proposer une redéfinition du rapport que Nabokov entretient avec le trivial dans son esthétique.

2 En mêlant références à la culture savante (beaux-arts, littérature, mythologie...) et citations de ce qu'il est convenu d'appeler la culture populaire ${ }^{3}$, Nabokov s'inscrit dans une tradition ancienne (comme le montrent les analyses de Rabelais menées par Bakhtine), mais aussi dans la tradition moderniste dans laquelle son art d'écrire s'est développé quand il était en Europe, de ses années d'études à Cambridge (1919-1922) jusqu'à son départ pour les États-Unis en 1940. Comme le note Andreas Huyssen, «modernism constituted itself through a conscious strategy of exclusion, an anxiety of 
contamination by its other : an increasingly consuming and engulfing mass culture " (Huyssen VII), et Nabokov pose un regard critique sur les images et le langage produit par la société de consommation américaine. Bien qu'indifférent (voire critique) à l'égard des expérimentations formelles d'un Duchamp ou du pop art, Nabokov s'inscrit pourtant dans la veine des artistes qui, notamment aux États-Unis, accordent une place très importante aux objets et systèmes de représentation de la vie quotidienne dans leur art, et, avec Lolita, il produit une œuvre souvent considérée par la critique comme son premier roman post-moderniste. Notons par ailleurs en préambule que, pour Nabokov, se déploie dans la culture populaire, et surtout dans les aspirations culturelles de la classe moyenne, ce qu'il appelle la poshlost, terme vaste que Sergej Davydov définit ainsi : "It refers to the broad range of cultural, social, and political phenomena under the category of "inferior taste". Nabokov elaborates on the concept in his book Nikolai Gogol (1944) "(Davydov 628). Nabokov définit la poshlost de la sorte : "Corny trash, vulgar clichés, Philistinism in all its phases, imitations of imitations, bogus profundities, crude, moronic and dishonest pseudoliterature - these are obvious examples " (Strong Opinions 101). Nabokov inclut de nombreux auteurs et artistes dans cette catégorie, qui ne se limite donc pas à la culture populaire, mais cette notion devait être rappelée pour traiter notre sujet.

Comme nous le verrons, Lolita présente plusieurs modes d'insertion de codes nonlittéraires, qui eux-mêmes relèvent de plusieurs techniques littéraires: la création d'effets de réel ${ }^{4}$, la parodie, l'intermédialité, la réappropriation des termes non littéraires dans les jeux de mots, l'utilisation des codes non-littéraires dans le complexe système de motifs et d'échos qui tisse la trame textuelle (en effet, les passages où Humbert Humbert cite ou parodie des textes non-littéraires sont souvent les lieux privilégiés de l'entrelacement de motifs importants). En outre, le plus souvent, ces divers modes d'insertion du texte non-littéraire sont associés, et même si les diverses parties de cette étude sont centrées sur l'une ou l'autre de ces techniques à des fins de démonstration, il est important de garder à l'esprit qu'elles sont mises en œuvre conjointement dans la trame textuelle. Grâce aux notes préparatoires au roman conservées à la Bibliothèque du Congrès, il s'agira de comparer la manière dont Nabokov combine le matériau non-littéraire à son écriture avec sa pratique intertextuelle plus traditionnelle, afin d'identifier la place et le rôle de ces références dans sa poétique.

\section{L'intertextualité comme outil de critique de la société de consommation}

4 Lorsque Nabokov s'est lancé dans la composition de Lolita, cela ne faisait que huit ans qu'il s'était installé aux États-Unis, et le milieu universitaire intellectuel où il évoluait en temps normal ${ }^{5}$ ne lui donnait pas beaucoup l'occasion de se plonger dans la culture qu'il avait besoin de recréer autour de son héroïne : un univers de sodas et sundaes consommés dans des roadside diners, de chansons sirupeuses de crooners jouées sur des juke-box, de conseils de beauté et de mode à caractère prescriptif, de publicités dont le message est pris pour argent comptant, d'idéal de vie calqué sur celui des stars d'Hollywood, dont on copie les tenues, coiffures, véhicules, manières de parler. Les premiers critiques du roman (eux-mêmes issus de l'élite intellectuelle du pays) ont très fréquemment mis l'accent sur le portrait de la classe moyenne américaine d'où est issue 
la protagoniste, allant jusqu'à voir dans l'œuvre non seulement une satire de la société américaine, mais également une œuvre anti-américaine (une accusation qui blessa profondément l'auteur $\left.{ }^{6}\right)$. En effet, tout comme Adorno ${ }^{7}$, Nabokov déplore la pauvreté esthétique et la niaiserie d'un certain nombre de productions culturelles dites de masse (pulp fiction, science-fiction, romances à l'eau de rose, comédies musicales) ${ }^{8}$, et se moque des aspirations intellectuelles de la classe moyenne en la parodiant.

5 L'insertion de référents textuels issus de la culture populaire par la parodie permet donc de critiquer la société de consommation des années 1950, et l'outil parodique est lui-même rendu particulièrement efficace par la structure narrative du roman. En effet, grâce au point de vue de son narrateur Humbert Humbert, qui se présente comme un intellectuel européen raffiné et donc choqué par la vulgarité du nouveau monde, l'auteur parvient à un subtil mélange intertextuel mêlant références littéraires canoniques et citations triviales, qui illustre combien Nabokov a su s'approprier ces codes nouveaux pour mieux les détourner. L'une des sources non-littéraires les plus importantes du roman sont les magazines, dont le rôle dans l'intrigue et dans le tissu textuel du roman est capital. L'objet de la présente analyse n'est pas de retracer toutes les occurrences du thème des magazines, mais nous pouvons brièvement énumérer les plus importantes d'entre elles. Rappelons tout d'abord que les magazines font partie intégrante du décor à 342 Lawn Street : ils sont en effet mentionnés dès les premières impressions que Humbert a des lieux-«But there was no question of my settling there. I could not be happy in that type of household with bedraggled magazines on every chair " (Lolita 37). Humbert utilise ensuite l'un de ces magazines comme prétexte pour pouvoir toucher Lolita lors de la scène où il se masturbe contre elle sur le divan du salon par un dimanche matin ensoleillé (chapitre 13, $1^{\text {ère }}$ partie) : les magazines sont donc logiquement présents dans la liste des "props " (Lolita 57)-les accessoires de cette scène répertoriés par Humbert dans la parodie de didascalies qui ouvre le chapitre. Notons aussi que c'est justement parce qu'elle descend s'acheter un magazine dans le hall de l'hôtel des Enchanted Hunters que Lolita rencontre Quilty (Lolita 138). Enfin, parmi les derniers objets lui restant de Lolita dont Humbert se débarrasse pour tenter de guérir son cœur brisé, figure " an accumulation of teen magazines » (Lolita 254). Toutefois, les magazines (qu'ils soient féminins, de décoration, destinés aux adolescentes ou consacrés aux stars d'Hollywood) ne sont pas que des accessoires utilisés pour faire progresser l'action du roman (comme dans la scène du chapitre 32 où Lolita rencontre Quilty parce qu'elle vient chercher un magazine), pour produire des effets de réel (la description de la maison de Charlotte), ou comme auxiliaires de caractérisation: leur langage et leurs images sont également finement intégrés à la trame textuelle du roman. Les magazines permettent ainsi de manière récurrente à Humbert de définir les standards culturels de la nymphette (qu'il méprise) : « Mentally, I found her to be a disgustingly conventional little girl. Sweet hot jazz, square dancing, gooey fudge sundaes, musicals, movie magazines and so forth - these were the obvious items in her list of beloved things » (Lolita 148, c'est moi qui souligne).

Nabokov s'approprie les codes linguistiques des magazines et invente par exemple des titres, dénonçant ainsi, par le jeu parodique, la naïveté du lectorat de ce type de publication : le magazine Glance and Gulp (Lolita 47) qui s'échange entre les habitants de la maisonnée Haze comme les livres d'une bibliothèque indique bien, par le biais de l'allitération, que tout ce qui y est aperçu est immédiatement gobé, avalé sans aucune forme de mise à distance. Nabokov utilise aussi de manière récurrente les guillemets 
pour insérer des fragments de textes non-littéraires, ce qui permet de placer ces citations à une distance ironique, et de souligner la différence entre le vocabulaire érudit et soigné du narrateur et le lexique publicitaire :

We came to know - nous connûmes, to use a Flaubertian intonation - the stone cottages under enormous Chateaubriandesque trees, the brick unit, the adobe unit, the stucco court, or what the Tour Book of the Automobile Association describes as "shaded" or "spacious" or "landscaped" grounds. (...)

But I did surrender, now and then, to Lo's predilection for "real" hotels. (...) Most tempting to her, too, were those "Colonial" Inns, which apart from "gracious atmosphere" and picture windows, promised "unlimited quantities of M-m-m food". (Lolita 146-147)

7 En-dehors de la conjonction de références littéraires et non-littéraires, on notera le recours aux guillemets, qui créent immédiatement une aura de suspicion autour des mots qu'ils accompagnent; ils agissent comme des agents de rupture du lien entre signifiant et signifié, car ils trahissent le caractère vide, mensonger de ces adjectifs commerciaux non mérités. Ce type d'interdiscursivité a donc un statut fort différent des citations littéraires dont Humbert émaille son manuscrit, et qui ont pour fonction de valider sa posture d'expert ${ }^{9}$, d'intellectuel, d'homme de goût en qui l'on peut avoir toute confiance. Il s'observe donc ici, par la citation de textes non-littéraires, une inversion de la fonction de la citation, qui devient ici l'objet de suspicion.

Une autre source textuelle non-littéraire que Nabokov a absorbée et intégrée à son roman provient des voyages que l'auteur fit à travers les États-Unis avec son épouse Véra pour chasser les papillons, lors desquels il séjourna dans de nombreux motels. Les notes préparatoires aux romans contiennent ainsi des copies de diverses instructions destinées aux clients (certaines copiées de la main de Véra), dont on retrouve la trace moqueuse dans le roman. Les notes préparatoires conservées à la Bibliothèque du Congrès comprennent cette citation inscrite par Véra : « do not throw rags in toilet; a waste basket is provided in your cabin for this purpose. Do not throw grease (?) in toilet $»^{10}$. Dans le roman, Nabokov recrée des instructions que cite Humbert :

Some motels had instructions pasted above the toilet (on whose tank the towels were unhygienically heaped) asking guests not to throw into its bowl garbage, beer cans, cartons, stillborn babies. (Lolita 146)

9 La liste qui occupe cet extrait s'apparente à un catalogue surréaliste, mais combine également un effet de réel (Barthes 82), du fait de la description méticuleuse, et une insinuation morbide qui renvoie à l'avant-propos de John Ray Jr. où le sort de Lolita, qui meurt en couches en donnant naissance à une enfant mort-née, est révélé obliquement. L'effet premier de moquerie de la part de Humbert l'Européen, qui a grandi dans un hôtel de luxe de la Côte d'Azur, se double donc d'effets analeptiques et proleptiques à visée structurelle. Notons qu'il s'agit là de caractéristiques structurelles partagées par la plupart des textes-dans-le-texte du roman (voir analyse de l'extrait du Who'sWho un peu plus loin), tels que la carte postale envoyée par Lolita, la lettre de Charlotte, la chanson de Carmen Barmen, le poème écrit après la disparition de l'héroöne, ou encore le papier en-tête des Enchanted Hunters.

Dans son entreprise parodique, Nabokov imite également la typographie de ces textes rencontrés le long des routes: " TIMBER HOTEL, Children under 14 Free » (Lolita 146). Cette citation produit un effet de réel et attire l'œil, pour mieux mettre en valeur le caractère économique de l'offre commerciale, en écho à la manière insistante qu'a le narrateur d'énumérer combien ces voyages lui coûtent, et combien le fait que Lolita soit si jeune 
est intéressant d'un point de vue pécuniaire-ce qui attise aussi son désir : «I derived a not exclusively economic kick from such roadside signs as TIMBER HOTEL, Children under 14 Free ", (Lolita 146, c'est moi qui souligne). La réification de Lolita en objet de consommation dans la société humbertienne de consommation n'en est que renforcée : un vaste réseau sémantique qui parcourt le roman ne cesse en effet de traiter Lolita comme un objet, un article dans un rayon ${ }^{11}$. Par un effet de miroir pervers, Lolita incarne à la fois la consommatrice idéale-" She it was to whom ads were dedicated : the ideal consumer, the subject and object of every foul poster " (Lolita 148)-et l'objet de consommation dont Humbert ne peut se passer. Il est donc possible de considérer l'omniprésence du langage commercial, pécuniaire et publicitaire au sein du texte poétique humbertien comme le signe de la transformation de Lolita en objet de consommation (concept de commodification). L'omniprésence du lexique publicitaire dans un texte qui se présente comme un chant d'amour peut être interprétée comme une forme d'intertextualité diffuse, voire systémique, car elle relève de la stratégie nabokovienne souterraine qui sape la rhétorique humbertienne et expose les aspects les plus insoutenables de la relation du narrateur à Lolita.

11 La critique de la société de consommation est donc d'autant plus subtile que son modèle de consommation sans frein est dupliqué par Humbert qui consomme sexuellement sans frein l'orpheline qu'il emmène de motel en motel. Nabokov procède de manière similaire en ce qui concerne sa critique de la culture de masse américaine, mais en alliant des dispositifs intertextuels et intermédiaux.

\section{Structure du roman et culture de masse}

12 Le système de la culture de masse qui relie la publicité, les conseils publiés dans les magazines, le culte des célébrités, et les objets qu'il faut consommer, n'est pas perçu par les personnages américains du roman (notamment Charlotte et Lolita), alors qu'il est clairement identifié et critiqué par Humbert Humbert :

She believed, with a kind of celestial trust, any advertisement or advice that that appeared in Movie Love or Screen Land - Starasil Starves Pimples, or "You better watch out if you're wearing your shirttails outside your jeans, gals, because Jill says you shouldn't". ${ }^{12}$ (Lolita 148)

13 D'une manière encore plus subtile, Nabokov se sert même du système qui régit la culture de masse et la société de consommation-la création de besoins chez le consommateur via la publicité qui utilise des célébrités en les érigeant en modèlespour la trame même de son roman, et notamment, pour tisser le motif de la présence de Clare Quilty (motif que le lecteur est précisément invité, par relecture, à retracer). En effet, la présence de Quilty dans le texte, très subtilement encodée et imperceptible à la première lecture, est presque systématiquement liée à la culture de masse américaine. La première occurrence complète de son nom et de sa profession se trouve dans un autre texte-dans-le-texte non-littéraire, le soi-disant extrait de l'édition de $1946 \mathrm{du}$ Who's Who in the Limelight, l'un des rares livres disponibles dans la prison depuis laquelle Humbert rédige son récit. Cet ouvrage recense les noms de ceux qui composent le monde de l'industrie du spectacle, parmi lesquels :

Quilty, Clare, American dramatist. Born in Ocean City, N.J., 1911. Educated at Columbia University. Started on a commercial career but turned to playwriting. Author of The Little Nymph, The Lady Who Loved Lightning (in collaboration with Vivian Darkbloom), Dark Age, The Strange Mushroom, Fatherly Love, and others. His 
many plays for children are notable. Little Nymph (1940) traveled 14,000 miles and played 280 performances on the road during the winter before ending in New York. Hobbies : fast cars, photography, pets. (Lolita 31)

14 Cette entrée du Who's Who abonde, à la deuxième lecture, en références qui indiquent que Quilty est bien celui qui a volé Lolita à Humbert : le titre de son grand succès The Little Nymph, la présence d'une homonyme de Lolita dans l'entrée suivante du dictionnaire, la mention de son acolyte Vivian Darkbloom, l'importance de ses pièces pour enfants, ses passe-temps qui font écho à la course-poursuite du deuxième périple des protagonistes, à ses pratiques photographiques perverses, et à Lolita elle-même, que Humbert appelle à de nombreuses reprises «my pet». Notons par ailleurs qu'il est indiqué ici que Quilty a d'abord fait carrière dans la publicité, ce qui constitue un point commun supplémentaire avec son double Humbert Humbert, dont la mère était en outre « une dame qui aimait les éclairs » / "A lady who loved lightning », puisqu'elle meurt foudroyée. L'encodage proleptique se poursuit dans le paragraphe qui suit immédiatement la citation ci-dessus. Humbert y illustre sa capacité à copier le code du Who's Who en inventant une entrée pour Lolita où se dessinent des références au meurtre du dramaturge (rappelons qu'à ce stade du roman le lecteur sait que Humbert est un meurtrier mais ignore toujours l'identité de sa victime) : «Born 1935. Appeared (...) in The Murdered Playwright. Quine the Swine. Guilty of killing Quilty. Oh, my Lolita, I have only words to play with! (Lolita 32). Si Humbert n'a que les mots avec quoi jouer, ce passage montre qu'il est déjà passé maître dans l'art de copier et parodier le langage du monde d'Hollywood.

Quilty est donc, dès sa première apparition dans la trame textuelle, établi comme une célébrité13 ${ }^{13}$ mais Nabokov ne se contente pas d'insérer de manière subtile le langage de ce monde des stars : l'auteur intègre également à son texte les images de la société du spectacle telles que la société de consommation les reproduit dans la publicité. Ainsi, la deuxième convocation de Quilty sur la scène du roman est faite de manière intermédiale, par le biais d'une image, celle de la publicité que Lolita a punaisée au mur de sa chambre: "A distinguished playwright was solemnly smoking a Drome. He always smoked Dromes. The resemblance was slight » (Lolita 69). Cette courte ekphrasis d'une publicité (fictive) est l'un des indices de l'identité du rival de Humbert, et elle joue un rôle structurel d'autant plus important que juste auparavant Humbert a décrit une autre publicité, celle du "Conquering Hero", publicité bien réelle retrouvée et reproduite par Alfred Appel dans les notes au roman (Lolita 369), dont le protagoniste ressemble effectivement à Humbert, et qui a dû amuser Nabokov car cette publicité cite un poème épique de Thomas Morell, et produit donc une conjonction de références high brow (savantes) et low brow (populaires). Les deux rivaux Humbert et Quilty, ou plutôt les deux faux frères, sont donc tous deux présentés de la même manière, via une ekphrasis peu conventionnelle, puisqu'il s'agit ici de décrire des publicités et non des œuvres d'art.

Lors de l'occurrence suivante du nom de Quilty, c'est à nouveau son statut de célébrité et de protagoniste d'une publicité qui est rappelé, lorsque Lolita le reconnaît dans la salle à manger des Enchanted Hunters :

"Does not he look exactly, but exactly, like Quilty?" said Lo in a soft voice, her sharp brown elbow not pointing, but visibly burning to point, at the lone diner in the loud checks, in the far corner of the room.

"Like our fat Ramsdale dentist?" (...)

"Course not," she said with a splutter of mirth. "I meant the writer fellow in the 
Dromes ad."

Oh, Fame ! Oh, Femina ! (Lolita 121) cigarette Drome ${ }^{14}$, en une sorte de fusion entre lui-même et le produit qu'il promeut, mais ce qu'il faut souligner ici c'est l'insistance de Humbert sur le lien entre les publicités de magazine, la célébrité, et le lectorat féminin de ces magazines: «Oh, Fame! Oh, Femina!", où l'on peut sans doute détecter un jeu de mots bilingue sur le mot «Fame» et «femme » quand «Fame» est prononcé à la manière française. Le roman montre en effet que les magazines féminins, qu'ils soient destinés aux adolescentes ou aux adultes, ou qu'ils soient plus spécifiquement consacrés aux stars de cinéma, jouent un rôle de premier plan dans la vie quotidienne, la culture, les goûts, le comportement et les aspirations des deux personnages féminins principaux du roman, Charlotte et Lolita. Les deux publicités arrachées à un magazine et affichées dans la chambre de Lolita représentent ainsi, de manière intermédiale, le lien entre son existence et celle des célébrités représentées sur papier glacé, tout en fournissant deux éléments essentiels de caractérisation, de développement de l'intrigue, et de structure.

Notons par ailleurs que Nabokov, dans sa première version du scénario de Lolita, avait prévu une courte séquence de préparation de ce passage important du roman, où l'on devait voir Lolita déchirant la publicité où figure Quilty: "The last issue of "File" lies on the turf near the deck chair. She contemplates the back cover, then rips it off and takes it upstairs to her room, leaving the rest of the magazine on the chair " $^{15}$. "File " est bien sûr une anagramme du célèbre magazine «Life », qui représente, par son titre même, les aspirations de toute une société à avoir une vie semblable à celle des stars ${ }^{16}$. Humbert ne cesse de rappeler combien le comportement de Lolita est lié à ce qu'elle lit dans les magazines: "I knew she would let me do so, and even close her eyes as Hollywood teaches » (Lolita 48), et comme l'a démontré Monica Manolescu, il se sert de la fascination de Lolita pour le monde des célébrités via son propre physique qui correspond aux canons masculins hollywoodiens (Manolescu 167-168) pour la séduire et la manipuler.

Dans la version finale du scénario, Nabokov a même ajouté un détail de la publicité pour les cigarettes Drome, le slogan/les pseudo-mots du célèbre dramaturge vantant les mérites de ces cigarettes: "I can write without a pen but not without a Drome" (Screenplay 72). Le ridicule du slogan rappelle des slogans similaires que Nabokov parodie ici, et qui étaient justement très fréquents dans les publicités pour les cigarettes Camel, pour lesquelles, à la fin des années 1940 et tout le long des années 1950, des dizaines d'acteurs, chanteurs, ou ténors d'opéra ont été utilisés selon un schéma visuel identique et dont voici quelques exemples : 


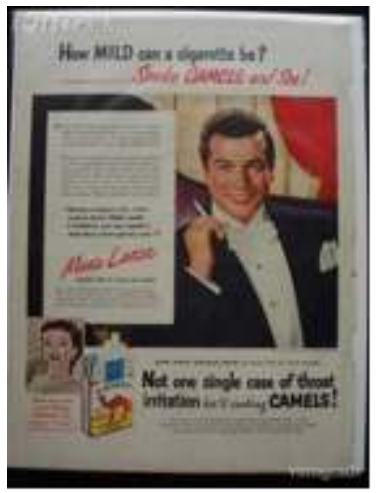

Camel ad, 1950
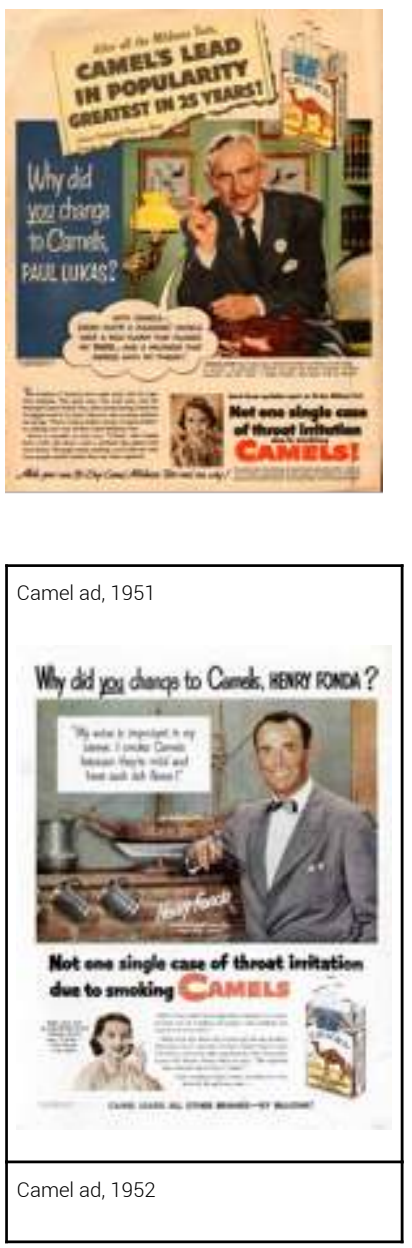

20 En faisant ainsi appel à la mémoire visuelle et à la culture populaire de son lecteur ${ }^{17}$, Nabokov parvient à introduire à la fois un jeu de mots visuel et linguistique sur le mot «Drome », qui rappelle le mot "dromedary» / dromadaire, une sorte de double simplifié d'un «camel » / chameau (le dromadaire comme chameau à une bosse), et indique aussi une incohérence que le naturaliste Nabokov n'avait pas manqué de remarquer, à savoir que le paquet de Camel est illustré par un dromadaire et non un chameau, comme le voudrait la logique, ainsi qu'Alfred Appel l'a noté.

21 En décrivant une publicité qu'il invente de toutes pièces, Nabokov duplique par la parodie le système de représentation publicitaire, et en dénonce subtilement la nature 
factice. En effet, en créant une fausse publicité, il rappelle le caractère mensonger de toutes les publicités, qui produisent des images destinées à faire croire au consommateur qu'elles font partie de son univers: les publicités tentent d'oblitérer leur propre processus de représentation afin de permettre une meilleure identification du consommateur avec le monde qu'elles promeuvent. Comme il l'avait énoncé dans son étude consacrée à Gogol et publiée en 1944, les publicités de magazine offrent un exemple parfait de poshlost : "The rich poshlust of advertisements of this kind is due (...) to suggesting that the acme of human happiness is purchasable and that its purchase somehow ennobles the purchaser » (Nicolai Gogol 67).

Les analyses précédentes donnent ainsi à voir une fonction de l'interdiscursivité nonlittéraire avant tout d'ordre dénonciateur, sur plusieurs plans, puisqu'elle permet non seulement de critiquer la société de consommation et la culture de masse, mais aussi de créer une trame souterraine qui dénonce les agissements de Humbert Humbert. Toutefois, le rapport qu'entretient Nabokov à son nouvel environnement américain est complexe, et les occurrences intertextuelles qui empruntent à des sources de la culture populaire ne sauraient être réduites à cette posture critique. Comme l'a énoncé Suellen Stringer-Hye: "Nabokov's own attitudes toward popular culture are problematic. He seems on the one hand to detest its vulgarity while on the other to celebrate its vigor " (Stringer-Hye 158). Le rôle de premier plan qu'il confie aux publicités de Quilty et Humbert dans le roman illustre la relation paradoxale que l'écrivain entretient avec le trivial ; dans sa postface à Lolita il affirme en effet : « Nothing is more exhilarating than philistine vulgarity » (Lolita 315).

\section{De la parodie au ré-enchantement, un autre aspect de l'intertextualité low brow du roman}

23 L'ambivalence de Nabokov à l'égard de ce qui relève de la poshlost se laissait déjà percevoir dans son ouvrage consacré à Gogol, dans lequel il regrettait tout autant la présence de la poshlost au fil des pages gogoliennes qu'il s'émerveillait de la capacité de l'auteur à transformer en chef-d'œuvre exquis une image des plus sordides ${ }^{18}$. Notre étude propose d'étudier à présent la manière dont les stratégies interdiscursives nabokoviennes procèdent à un ré-enchantement des référents vulgaires empruntés à la culture populaire par une appropriation des codes linguistiques non-littéraires qui permet d'en révéler le potentiel poétique.

Comme cela a été vu plus haut, Nabokov se montre très habile à parodier et créer des publicités et des slogans, et le narrateur de son roman illustre la même habileté à manipuler les codes de la publicité : malgré son statut d'universitaire, nous apprenons aussi qu'Humbert Humbert, issu d'une famille de commerçants, a lui-même travaillé dans la publicité pour parfums :

In New York I eagerly accepted the soft job fate offered me: it consisted mainly of thinking up and editing perfume ads. I welcomed its desultory character and pseudoliterary aspects, attending to it whenever I had nothing better to do. (Lolita 32)

Parmi les envolées lyriques, les jeux de mots et les références littéraires qui caractérisent la prose de Humbert, ce dernier glisse donc un certain nombre d'expressions directement tirées de la publicité. Par exemple, pour désigner la proposition de mariage que lui a faite Charlotte, il utilise l'expression « the Amazing 
Offer (Lolita 71) »-expression dont les majuscules rappellent immédiatement celles des slogans destinés à faire succomber le client par des offres sans pareille. De même, lorsqu'il s'agit de donner à Lolita les somnifères qu'il a essayés sur Charlotte et dont il espère que la puissance lui permettra de caresser Lolita à son insu, il emploie naturellement le langage publicitaire auquel Lolita est si sensible. Après avoir sorti les « Purple Pills » de leur boîte, il en fait l'article, tel un bon agent commercial :

"Blue!" she exclaimed. "Violet blue. What are they made of?"

"Summer skies," I said, "and plums and figs, and the grapeblood of emperors."

"No, seriously - please."

“Oh, just Purpills. Vitamin X. Makes one strong as an ox or an ax. Want to try one?"

Lolita stretched out her hand, nodding vigorously. (Lolita 122)

On remarquera ici la manière dont Nabokov mêle le langage commercial et poétique. Les images lyriques se succèdent (mais manquent de convaincre Lolita), les allitérations font vibrer la surface des mots, les jeux de mots déploient les potentiels du signifiant ("purpills", "strong as an ox or an ax»), tout en mimant la syntaxe typique des slogans publicitaires, qui procède à l'élision du sujet de la phrase, et en introduisant du lexique prétendument scientifique qui achève de gagner la confiance de la nymphette qui tend la main. Une telle hybridité langagière qui mêle la syntaxe et le lexique caractéristiques du langage publicitaire aux traits stylistiques de la poésie trouve son point de rencontre dans le système allitératif à l'œuvre ici, puisque le recours aux allitérations est un signe distinctif commun aux codes publicitaire et poétique.

La manière dont Nabokov composa son texte révèle cette hybridité intrinsèque : une comparaison des sources non-littéraires de Lolita au texte final nous permet de davantage percevoir comment cette interdiscursivité mixte, ou trans-classe culturelle, s'est construite. Pour écrire Lolita, Nabokov s'est largement documenté sur de nombreux sujets qu'il ne maîtrisait pas (comme le fonctionnement d'un revolver ou l'évolution anatomique des fillettes), et il a également pris de nombreuses notes sur des éléments de la société de consommation américaine qu'il découvrait encore. Le manuscrit du roman, rédigé sur des index cards ${ }^{19}$ (fiches bristol) a été brûlé par Nabokov, bristol après bristol, dès que son épouse en avait tapé un à la machine, car dans les années 1950 l'auteur, craignant pour son poste à Cornell, envisageait encore de publier le roman de manière anonyme (Boyd 2011, 27) et redoutait sans doute de conserver un tel récit homodiégétique écrit de sa main. Il ne reste donc que les notes préparatoires au roman, qui sont conservées à la Bibliothèque du Congrès, mais cellesci sont de toute première importance pour cette analyse croisant critique génétique et études intertextuelles. Elles contiennent en effet de nombreuses citations extraites de divers magazines pour adolescentes (Miss America, Movie Teen), de publications spécialisées dans la vie des stars de Hollywood (Movie Love, Screenland), ou encore de magazines féminins, ouvrages ou catalogues de décoration. Certaines de ces notes ont été plus ou moins directement citées dans Lolita, et nous proposons à présent d'examiner plusieurs exemples de la manière dont Nabokov a inséré ces textes nonlittéraires dans son roman afin de savoir si ce matériau moins "noble» que les références littéraires ou artistiques est traité de manière spécifique par l'auteur. Le premier exemple est tiré d'une index card intitulée "17, sept. 1952 [on menstruation] ", sujet que l'on imagine peu connu de Nabokov, qui en outre n'a pas eu de fille mais un fils. Sur ce petit bristol, Nabokov a recopié plusieurs éléments, et l'on voit qu'il a déjà adapté ses notes à une possible utilisation dans le roman : 
Greedy as a young robin you started snatching up... crumb of information... about how they [older girl friends] had just "fallen off the roof" or how "Grandma was visiting". Probably, though we hope not, you heard references to "the curse" ... Then, on the morning before our thirteenth birthday, it happened We were, simply, menstruating. When will my uterus, asked Lo, start to build a thick soft wall on the chance that a possible baby may have to be bedded down there (... ${ }^{20}$.

Toutefois, dans le texte final, et contrairement à ce que suggère la fin des notes, l'auteur n'a pas repris la parcelle de dialogue entre adolescentes griffonnée sur ce bristol en attribuant ces paroles à Lolita, mais a intégré ces extraits de magazine à la voix de Humbert, en y adjoignant d'autres codes linguistiques :

Has she already been initiated by mother nature to the Mystery of the Menarche? Bloated feelings. The Curse of the Irish. Falling from the roof. Grandma is visiting. "Mr. Uterus [I quote from a girls' magazine] starts to build a thick soft wall on the chance a possible baby may have to be bedded down there." The tiny madman in his padded cell. (Lolita 47)

30 Certaines caractéristiques stylistiques identifiées plus haut sont à nouveau mobilisées ici (les allitérations, l'utilisation des majuscules, l'insertion d'une citation entre guillemets), mais nous souhaiterions souligner ici la juxtaposition de clichés («mother nature »), de métaphores populaires directement empruntées au magazine pour adolescentes, du procédé infantile de personnification «Mr. Uterus», et du terme scientifique médical "Menarche » désignant la première menstruation (ce terme ne faisant pas partie du langage courant, Alfred Appel élucide son sens dans une note). Le texte-source initial est donc à la fois parodié, complexifié, densifié, enflé en quelque sorte tout comme ces "sensations de ballonnement» («bloated feelings ») évoquées ici. Un certain malaise est produit par cet enchaînement de prose savante et de langage immature, sans doute destiné à attirer l'attention du lecteur sur le sujet même de ces phrases, qui pose une question capitale : Lolita est-elle toujours une enfant, ou est-elle devenue une femme fertile? L'hybridité nauséeuse des codes est en outre comme dupliquée dans le jeu polysémique très particulier produit par l'image du mur capitonné («a thick soft wall») extraite des magazines recopiés, car cette métaphore est ici reliée à une référence aux séjours en hôpital psychiatrique de Humbert, ce qui établit, de manière assez morbide, un parallèle entre l'utérus de Lolita et une cellule où l'on enferme les aliénés. De la sorte, le désir de Humbert pour le corps de Lolita, et notamment ses organes sexuels (« My only grudge against nature was that I could not turn my Lolita inside out and apply voracious lips to her young matrix » Lolita 165) est une fois de plus subtilement mis en parallèle avec la folie et la perversion du narrateur ${ }^{21}$. L'on remarquera que lorsqu'Humbert déploie les allusions intertextuelles à Poe, Dante ou Ronsard, un malaise de ce type n'est pas suscité : c'est précisément la juxtaposition de codes savants et populaires qui permet de saper la rhétorique humbertienne.

31 Cet exemple illustre donc le travail littéraire effectué à partir des matériaux nonlittéraires collectés dans les magazines que l'auteur a lus, mais présente également d'autres caractéristiques du style nabokovien : le goût de Nabokov pour les mots rares, sa manie des allitérations, la récurrence des jeux de mots et le choix de métaphores qui font écho à un motif récurrent du roman et contribuent à en tisser la trame (ici, l'état mental du narrateur et le crime qu'il s'apprête à commettre). Ce travail à partir des magazines féminins et des magazines pour adolescentes a permis à Nabokov de se familiariser avec les intérêts de ses nouvelles compatriotes, et surtout, avec leur 
langage, notamment dans le but de créer des personnages aussi vraisemblables que possible, confirmant ainsi l'idée de Christine Raguet selon laquelle l'illusion de réalité s'ancre dans le langage (Raguet-Bouvart 208). Comme l'a expliqué Philippe Hamon, « ce n'est jamais, en effet, le "réel" que l'on atteint dans un texte, mais une rationalisation, une textualisation du réel, une reconstruction a posteriori encodée par et dans le texte [...]» (Hamon 129). Dans Lolita, cette textualisation du réel passe par le dispositif intertextuel, à mesure que Nabokov intègre à la fiction des éléments tirés de textes non-littéraires. Les citations qui suivent reproduisent les notes de Nabokov reprenant des éléments de deux magazines, Miss America et Movie Love :

I Miss America

November (acquired August 30) 10 cents

"Atlas" comics. "The Best in Teen Tales"

"Don't be fat" ads, one of them urging the purchase of "the exciting new TUMMY

FLATTENER [exciting what?]

Patsy Walker [change to something else]

Nossir Daddums

[Dad in purple suit forbidding shoulder-length red-head to see school (or college)

chum who writes poetry for her. Her tears about the size (and exactly same outline)

as her small brother's freckles only more oval

II Miss America (cont.)

"Atlas" [change this]

"Learn to draw" ad

Another Patsy Walker episode

[they are "students". Apparently high school but look like college girls and boys]

[check meaning of word "dud" its origin, etc.]

III Miss America Nov. 1952

Ad: Need extra spending money?

[selling samples of gorgeous Christmas cards]

Some more Patsy W. and Hedy Wolfe (the bad girl dark hair, fat father with cigar

whereas Mr W. has little moustache and good looking)

(...)

Yet another shape ad. Front zipper that trim tummy, nips hips.

I Movie Love Oct. (Aug. 30) 10 cents

Ad: Reduce your appearance instantly, girdle: creates slimmer figure that invites romance

$(\ldots)^{22}$

Toutes ces citations tirées de Miss America et Movie Love ont été utilisées par l'auteur pour composer le passage suivant, qui semble nous faire feuilleter ces magazines grâce à une syntaxe resserrée, produite par l'accumulation de phrases courtes, voire nominales, qui font référence à des choses très diverses, sans lien entre elles (ainsi que le soulignent les points qui séparent les phrases de manière incisive), et qui produisent un effet catalogue :

One day I removed from the car and destroyed an accumulation of teen-magazines.

You know the sort. (...) Invite Romance by wearing the Exciting New Tummy

Flattener. Trims tums, nips hips. Tristram in Movielove. Yessir! The Joe-Roe marital enigma is making yaps flap. Glamorize yourself quickly and inexpensively. Comics. Bad girl dark hair fat father cigar; good girl red hair handsome daddums clipped mustache. Or that repulsive strip with the big gagoon and his wife, a kiddoid gnomide. Et moi qui t'offrais mon génie... (Lolita 254)

33 Comme le souligne Yannicke Chupin, cette liste "anarchique, parataxique et allitérative » « fait écho à la rapidité et à l'efficacité de la publicité américaine », elle est composée de « fragments visuels et linguistiques qui sont pour lui le reflet de la culture 
de masse américaine" (Chupin 55), et qui par l'absence de coordination et de subordination permet de juxtaposer les éléments sans les hiérarchiser (Chupin 56). Si l'on peut, comme Yannicke Chupin, voir dans ce choix syntaxique un moyen d'opposer la langue américaine, cette "langue du slogan, une langue qui doit son staccato caractéristique à l'accumulation de monosyllabes » (Chupin 55) à la langue française et ses circonvolutions syntaxiques dans des phrases interminables, on peut aussi interpréter la structure syntaxique de l'extrait ci-dessus comme l'équivalent verbal de l'action de feuilleter l'un de ces magazines pour adolescentes, en écho au geste que Nabokov a lui-même effectué pour prendre ces notes. En effet, contrairement à ce que pressent Yannicke Chupin, peut-être du fait de l'absence de guillemets, Humbert ne « feint » pas « de recopier différents magazines » (Chupin 55) : ainsi qu'en attestent les notes préparatoires au roman, il s'agit ici de fragments véritablement recopiés de magazines réels, que Nabokov recombine pour produire à la fois un effet de réel, une parodie, mais aussi, comme le voit Rachel Bowlby, un hommage à la poésie produite par la publicité (Bowlby 177): le lecteur ne peut qu'être impressionné par le vertige allitératif et assonantique de ce passage, la virevolte de clins d'œil intertextuels (Sterne, Tristan et Iseult ${ }^{23}$, Chateaubriand, Musset), l'explosion néologique évoquant les personnages de Dick Tracy et Kerry Drake par Alfred Andriola ${ }^{24}$ : "gagoon », qui fait résonner "gag», "goon» et «baboon»; «kiddoid», adjectif dont le suffixe -oid indique qu'il signifie " ayant l'aspect d'un enfant »; " gnomide » qui mêle le gnome et le bromure (bromide en anglais). Notons enfin le jeu intermédial ouvert par les deux derniers fragments, où l'on est visuellement saisi par la force évocatrice de la courte ekphrasis sans prédicat qui reprend les caractéristiques de la bande dessinée Patsy Walker notées par Nabokov et oppose les deux personnages principaux, Patsy Walker et Hedi Wolfe grâce à un parallèle syntaxique parfait : « Bad girl dark hair fat father cigar ; good girl red hair handsome daddums clipped mustache ».

La manière nabokovienne de rendre littéraire un matériau textuel et iconique initialement étranger à la prose poétique éminemment érudite de l'auteur s'illustre également dans une parodie à double niveau, littéraire et non-littéraire, développée par Humbert à des fins de critique de la société de consommation et de moquerie de Charlotte-deux stratégies qui visent à valoriser l'image du narrateur aux yeux du lecteur. Le narrateur consacre ainsi un long passage de son récit aux efforts déployés par Charlotte pour redécorer sa maison après leurs noces. Ce faisant, Humbert s'applique à suivre un topos littéraire bien connu-la demeure d'un personnage est un portrait oblique de celui qui l'occupe-mais Nabokov souligne subtilement ce topos grâce au titre de l'ouvrage (inventé) dont Charlotte suit les prescriptions : Your Home Is You. En fait, dans ce passage, le cliché littéraire est complètement subverti, car du fait des prescriptions de la société de consommation, Charlotte décore sa maison non pas selon ses goûts et sa personnalité, mais selon la mode et les standards de l'époque. On notera ainsi dans le passage suivant le champ lexical de la loi ou de la religion qui soulignent l'absence de décision libre de la part de Charlotte :

She rearranged the furniture - and was pleased when she found, in a household treatise, that "it is permissible to separate a pair of sofa commodes and their companion lamps." With the authoress of Your Home Is You, she developed a hatred for little lean chairs and spindle tables. She believed that a room having a generous expanse of glass, and lots of rich wood paneling was an example of the masculine type of room, whereas the feminine type was characterized by lighter-looking windows and frailer woodwork. The novels I had found her reading when I moved in were now replaced by illustrated catalogues and homemaking guides. (Lolita 78) 
De manière symptomatique, le remplacement des romans par des catalogues et des guides de décoration reflète non seulement l'invasion des objets de la société de consommation dans l'espace domestique, mais surtout l'invasion du lexique consumériste dans l'espace textuel, qui utilise l'effet catalogue, les répétitions (doublées d'allitérations), et les mots mêmes de ces catalogues :

With the zest of a banal young bride, she started to "glorify the home." Knowing as I did its every cranny by heart (...) I had long entered into a sort of emotional relationship with it, with its very ugliness and dirt, and now I could almost feel the wretched thing cower in its reluctance to endure the bath of ecru and ocher and putty-buff-and-snuff that Charlotte planned to give it. She never got as far as that, thank God, but she did use up a tremendous amount of energy in washing window shades, waxing the slats of Venetian blinds, purchasing new shades and new blinds, returning them to the store, replacing them by others, and so on, in a constant chiaroscuro of smiles and frowns, doubts and pouts. She dabbled in cretonnes and chintzes; she changed the colors of the sofa (...). (Lolita 77)

Humbert emprunte ici les termes mêmes des catalogues édictant la mode de l'époque, durant laquelle la tendance était à l'écru, comme l'indique le camaïeu de beiges introduit par la métaphore humoristique du bain («the bath of ecru and ocher and putt-buff-and-snuff»). Les noms de couleur que l'on trouve dans les catalogues ou les nuanciers commerciaux sont un élément récurrent des notes préparatoires au roman : Nabokov a souvent noté des noms de couleurs de divers biens de consommation (vêtements, voitures...) en y ajoutant certains de son invention qui révèlent le potentiel poétique du langage commercial. La virtuosité avec laquelle Nabokov manipule les codes de ce langage trouve une déclinaison remarquablement poétique dans la liste des couleurs de maillots de bain à la disposition d'Humbert dans le magasin aquatique : "Swimming suits? We have them in all shades. Dream pink, frosted aqua, glans mauve, tulip red, oolala black» (Lolita 107). Contrairement à d'autres passages étudiés, cette palette de couleurs ne résonne pas d'allitérations ni d'assonances, mais est plutôt d'une variété sonore qui duplique celle des modèles parmi lesquels le narrateur peut faire son choix. Les métaphores qui définissent ces couleurs jouent également sur des registres très variés : image glacée, métaphore florale, couleur abstraite du rêve, le savoureux « oolala black » où se lisent le français « ouh la la... » et un mélange anagrammatique de Lo et de Lola, mais aussi, dissimulé derrière le lexique anatomique savant, un clin d'œil érotique : le mot "glans", selon les notes érudites d'Alfred Appel, renvoie au " conical vascular body which forms the extremity of the penis » (Lolita 374).

Dans l'extrait consacré à la rénovation intérieure orchestrée par Charlotte, Nabokov s'amuse également à reprendre, cette fois sans les saper par un commentaire méprisant ou une métaphore comique, les principes de décoration qui reflètent les clichés de l'époque sur le genre via un parallèle lexico-syntaxique en chiasme qui permet d'opposer les deux sexes : " a room having a generous expanse of glass, and lots of rich wood paneling was an example of the masculine type of room, whereas the feminine type was characterized by lighter-looking windows and frailer woodwork». La présence de ce lexique commercial dans le texte littéraire est un élément caractéristique de Lolita: en effet, les autres romans et nouvelles de la période américaine de l'auteur ne sont pas autant nourris de cette observation de son pays d'adoption ni de sa découverte de la langue anglaise (ou plutôt américaine) nonlittéraire. Cette hybridation entre langue populaire et langue poétique sert plusieurs objectifs et produit plusieurs effets conjoints : elle manifeste par la parodie le goût de 
l'auteur pour le jeu sur les codes et sur le langage, tout en permettant à la fois une critique de la société de consommation et un émerveillement devant la multiplication d'objets et surtout de mots pour dire ce monde en prolifération constante. Rappelons en outre que la parodie est aussi un outil métatextuel puissant, comme le souligne Linda Hutcheon : "Parody is one of the major forms of self-reflexivity (Hutcheon 2). L'hybridation des codes littéraires et non-littéraires sert donc aussi le réflexe postmoderne d'attention au texte lui-même, et au geste recréateur du monde de l'écrivain : " [the creative artist] must possess the inborn capacity not only of recombining but of recreating the given world. In order to do this adequately, avoiding duplication of labor, the artist should know the given world" (Strong Opinions 32). Le travail de documentation et de recherche entrepris par Nabokov pour recréer le monde de son héroïne qu'il découvrait atteste de ses efforts pour connaître ce monde, et la confrontation des quelques notes de travail exposées dans cette étude nous permet d'entrevoir la manière dont Nabokov a entrelacé les mots et images qu'il découvrait dans son nouvel environnement et le registre poétique majeur qui est le sien.

Il reste encore de nombreuses sources à identifier et des dispositifs à analyser, mais il paraît important de souligner la part essentielle que joue ce matériau non-littéraire dans le développement de la langue littéraire américaine de Nabokov. La variété des registres que permet le jeu intertextuel nabokovien produit une ouverture du signifiant littéraire vers d'autres codes, tout en illuminant des aspects de la banale quotidienneté sous le faisceau de la littérature. Lolita démontre qu'il n'y a pas nécessairement concurrence entre culture de masse et littérature dans l'esthétique nabokovienne, contrairement à ce qui a pu être vu par certains critiques, qui de la sorte ont suivi la perspective humbertienne et ont condamné comme lui Lolita, la société américaine et les objets et textes de son quotidien par comparaison à la "noble » littérature et aux "beaux» arts. Notons toutefois qu'une tendance plus récente de la critique nabokovienne tend à renverser cette perspective ${ }^{25}$, telle Rachel Bowlby, qui voit dans la culture de masse la force vive de l'écriture du roman:

the driving force of the novel's language (...) is not so much its recognizable continuation of a literary tradition as its incorporation into the mass-cultural modes that make up Lolita's American world. (Bowlby 172)

Le roman embrasse en fait la nature ambivalente de l'héroïne qui lui donne son titre. Car si Lolita est le parfait reflet de la culture dans laquelle elle grandit ${ }^{26}$, elle suscite à la fois un désir brûlant et une forme de mépris chez Humbert, qui explique même que sa vulgarité, dérivée des publicités et des magazines, est justement l'une des conditions pour être une authentique nymphette: «What drives me insane is the twofold nature of this nymphet - of every nymphet, perhaps; this mixture in my Lolita of tender dreamy childishness and a kind of eerie vulgarity, stemming from the snub-nosed cuteness of ads and magazine pictures » (Lolita 44, c'est moi qui souligne) ${ }^{27}$. La beauté de l'héroïne, que le roman célèbre du premier au dernier mot, vouant Lolita à l'éternité des pigments picturaux, est celle de cette Amérique vulgaire et la poshlost, que Nabokov, sur les traces de Gogol, rend merveilleuse sous sa plume de poète et son regard de peintre ${ }^{28}$.

Comme le rappelle Bruce Bégout dans son ouvrage intitulé Lieu Commun, et comme l'ont perçu Emerson et Thoreau « la culture américaine s'est édifiée en fonction d'un intérêt essentiel pour la quotidienneté » (Bégout 15), la vie de tous les jours, "the near, the low, the common ${ }^{29}$, et Nabokov, en adoptant la langue américaine pour sa création littéraire et les États-Unis comme espace où poser son regard toujours en éveil, s'inscrit 
pleinement dans cette tradition, tout en l'enrichissant de la perspective lumineuse d'un étranger nouvellement arrivé :

Se tenant dans une position de dedans-dehors, sur le seuil même de la familiarité, ni trop proche ni trop loin, [l'étranger ou le voyageur] est l'homme qui peut nous apprendre de quoi le quotidien est réellement fait. (Bégout 2005, 38)

En entrelaçant textes et images du quotidien à sa langue littéraire éminemment riche, Nabokov permet en retour d'illuminer « une part de cette vie courante [qui] se dérobe du fait même de sa surprésence » (Bégout 2002, 12). La forme d'interdiscursivité qu'il a développée dans Lolita a permis de mettre les outils intertextuels (parodie, citation, pastiche) et littéraires (jeux de mots, effets sonores, stylistiques, syntaxiques, métaphoriques) au service d'une poétique du vulgaire et du banal, tant dans ses objets que ses mots-un manifeste esthétique qu'il l'énonçait dès 1925 dans sa nouvelle « $\mathrm{A}$ Guide to Berlin » :

I think that here lies the sense of literary creation: to portray ordinary objects as they will be reflected in the kindly mirrors of future times; to find in the objects around us the fragrant tenderness that only posterity will discern and appreciate in the far-off times when every trifle of our plain everyday life will become exquisite and festive in its own right: the times when a man who might put on the most ordinary jacket of today will be dressed up for an elegant masquerade. (Stories 157, c'est moi qui souligne).

\section{BIBLIOGRAPHIE}

Adorno, Theodor W., \& Max Horkheimer. « The Culture Industry : Enlightenment as Mass Deception ». Trans. John Cumming. The Critical Tradition : Classic Texts and Contemporary Trends. Boston : Bedford/St. Martin's, 2007. 1255-62.

Barthes, Roland. « L'effet de réel ». In Littérature et réalité. Paris : Seuil, « Points », 1982. 81-90.

Bégout, Bruce. Lieu Commun : Le motel américain. Paris : Allia, 2002.

Bégout, Bruce. La découverte du quotidien. Prolégomènes à une phénoménologie du monde de la vie. Paris : Allia, 2005.

Bowlby, Rachel. «Lolita and the Poetry of Advertising. » In Vladimir Nabokov's Lolita : A Casebook. Ed. Ellen Pifer. Oxford : Oxford University Press, 2002. 155-179.

Boyd, Brian. « Lolita : What We Know and What We Don't.» Cycnos : 24, 1 (2007) : 215-246.

---. Stalking Nabokov. New York : Columbia University Press, 2011.

Chupin, Yannicke. « "Do You Mind Very Much Cutting Out The French ?” Le français de Humbert dans Lolita de Vladimir Nabokov ». Revue française d'études américaines : 115 (2008) : 50-59.

Consulted May 30, 2016.

www.cairn.info/revue-francaise-d-etudes-americaines-2008-1-page-50.htm.

Compagnon, Antoine. La Seconde main ou le travail de la citation. Paris : Seuil, « Point », 2016.

Davydov, Sergej. « Poshlost ». In The Garland Companion to Vladimir Nabokov. Ed. Vladimir

Alexandrov. New York : Garland Publishing Inc, 1995. 628-32. 
Emerson, Ralph Waldo. " An Oration delivered before the Phi Beta Kappa Society, at Cambridge, August 31, $1837 »$. In Nature; Addresses and Lectures (1849).

Hamon, Philippe. «Un discours contraint ». In Philippe Hamon et al. Littérature et réalité. Paris : Seuil, 1982. 119-181.

Huyssen, Andreas. After the Great Divide : Modernism, Mass Culture, Postmodernism. Bloomington : Indiana UP, 1986.

Malcolm, Donald. « Lo, the Poor Nymphet » The New Yorker (November 8, 1958) : 195-199.

Manolescu, Monica. Jeux de mondes : l'ailleurs chez Vladimir Nabokov. Bordeaux : Presses Universitaires de Bordeaux, 2011.

Nabokov, Vladimir. Lolita : A Screenplay. New York : Vintage, 1994.

---. Nikolai Gogol. Norfolk, Conn. : New Directions Books, 1944.

---. Strong Opinions. New York : McGraw-Hill, 1973.

---. The Annotated Lolita. Preface and notes by Alfred Appel Jr.. New York : Vintage, 1995.

---. The Stories of Vladimir Nabokov. Ed. Dmitri Nabokov. New York : Vintage, 2002.

Niegaard, Ole. « Uncle Gustave is Present : The Canine Motif in Lolita ». Nabokov Studies : 9 (2005) : 133-155.

Raguet-Bouvart, Christine. « Lolita - Mon Amérique... ». ACRAA : 20 (novembre 1995) : 207-219.

Roper, Robert. Nabokov in America, On the Road to Lolita. New York : Bloomsbury, 2015.

Rothstein, Eric. « Lolita : Nymphet at Normal School ». Contemporary Literature : 41.1 (2000) :

22-55.

Schickel, Richard. «A Review of a Novel You Can't Buy ». The Reporter (November 28, 1957): 45-47.

Schuman, Sam. « On the Road to Canterbury, Liliput and Elphinstone - The Rough Guide : Satiric Travel Narratives in Chaucer, Swift and Nabokov ». Consulted May 30, 2016.

https://www.libraries.psu.edu/nabokov/schuman2.htm

Stringer-Hye, Suellen. « Vladimir Nabokov and Popular Culture ». In Discourse \& Ideology in Nabokov's Prose. Ed. David H. J. Larmour. New York : Routledge, 2002.

Wood, Michael. « Lolita revisited ». New England Review : Middlebury Series : 17.3 (1995) : 15-13.

\section{NOTES}

1. Sur la base des notes de Carl Proffer (Keys to Lolita), intégrées ensuite dans l'appareil critique et annotatif de Alfred Appel Jr., d'autres travaux sont régulièrement publiés dans The Nabokovian, Nabokov Studies ou sur le forum Nabokv-L et identifient d'autres motifs ou références intertextuelles au sein du roman.

2. Par exemple, Donald Malcolm ou Richard Schickel (cf. bibliographie).

3. La notion de culture populaire « souffre à l'origine d'une ambiguïé sémantique » (Cuche 79), née des différentes définitions possibles du mot "culture ", entendu soit au sens large comme ensemble des manifestations d'une société humaine donnée (rituels, langage, habitus, productions, etc) ou au sens plus restrictif d'ensemble des productions de type artistique ou de divertissement (littérature, musique, peinture, cinéma, etc). Nous entendrons l'expression 
«culture populaire» dans sa première acception, qui permet non seulement d'inclure la deuxième définition, mais correspond aux aspects du roman qui seront étudiés ici, puisque notre étude s'attache à tous les aspects populaires (par opposition à l'élite) de la société américaine d'après 1945. Pour désigner cette culture spécifique qui émerge dans un contexte d'expansion économique faisant émerger la société de consommation, la critique nabokovienne américaine utilise également le terme un peu plus restrictif de "culture de masse » (Appel, Bowlby) pour désigner les éléments de la culture matérielle, visuelle ou textuelle qui relèvent du quotidien, de la consommation, des objets de la vie courante. Nous utiliserons également ce terme lorsque nos analyses prendront pour objet des éléments directement liés à la consommation de masse.

4. Cf. Barthes, 81-90.

5. Il le décrit ainsi dans l'un de ses entretiens : «I came to America in 1940 and decided to become an American citizen, and make America my home. It so happened that I was immediately exposed to the very best in America, to its rich intellectual life and to its easygoing, good-natured atmosphere. I immersed myself in its great libraries and its Grand Canyon. I worked in the laboratories of its zoological museums. I acquired more friends than I ever had in Europe. My books - old books and new ones - found some admirable readers » (Strong Opinions 26).

6. Voir la postface au roman, « On A Book Entitled Lolita (Lolita 315), ou sa déclaration : « Well, I can only repeat that I have neither the intent nor the temperament of a moral or social satirist. Whether or not critics think that in Lolita I am ridiculing human folly leaves me supremely indifferent. But I am annoyed when the glad news is spread that I am ridiculing America " (Strong Opinions 25). Il affirma par ailleurs : « Satire is a lesson, parody is a game » (Strong Opinions 75).

7. Dans son essai co-écrit avec Max Horkheimer « The Culture Industry: Enlightenment as Mass Deception » publié en 1947 Adorno développe une analyse acerbe de la structure de la culture de masse qui produit à la fois les produits et les consommateurs de ces produits culturels populaires, et dépeint la culture de masse comme une force dégradante, totalisante et par-dessus tout uniformisante (1255-1258).

8. Par exemple: «I loathe popular pulp, I loathe gogo gangs, I loathe jungle music, I loathe science fiction with its gals and goons, suspense and suspensories. I especially loathe vulgar movies - cripples raping nuns under tables, or naked-girl breasts squeezing against the tanned torsos of repulsive young males » (Strong Opinions 117).

9. Comme le rappelle Antoine Compagnon, les citations que produit un auteur «sont nécessaires afin de qualifier le sujet (son "esprit" et non immédiatement son discours), de montrer qu'il a lu, qu'il sait, c'est-à-dire qu'il a le droit de prendre la parole » (Compagnon 318).

10. Library of Congress, Washington D.C., Manuscript Division. Vladimir Vladimirovich Nabokov Papers, Lolita, Index cards (unnumbered). Notes in English (1950-1955), container 11, folder 6 of 6. 11. La réification de Lolita est un procédé récurrent dans le roman, mais nous voudrions attirer l'attention sur l'expression «fast article » pour la désigner, qui fait retour à deux reprises dans les notes préparatoires au roman, et qui est utilisée directement dans le roman par Lolita ellemême : "You must be confusing me with some other fast little article » (Lolita 222). Library of Congress, Washington D.C., Manuscript Division. Vladimir Vladimirovich Nabokov Papers, Lolita, Index cards (unnumbered). Notes in English (1950-1955), container 11, folder 4 of 6.

12. Citation précisément tirée, selon les notes de Nabokov, du numéro de Screenland de Sept 1952, avec une modification onomastique : «Loretta Young » est devenue « Jill ». Library of Congress, Washington D.C., Manuscript Division. Vladimir Vladimirovich Nabokov Papers, Lolita, Index cards (unnumbered). Notes in English (1950-1955), container 11, folder 4 of 6.

13. Dans l'occurrence suivante, Charlotte le présente comme une référence évidente que Humbert devrait connaître, lorsqu'elle mentionne son dentiste : « Dr. Quilty. Uncle or cousin, I think, of the playwright » (Lolita 64).

14. " "Quilty," I said. "I want you to concentrate. You are going to die in a moment. The hereafter for all we know may be an eternal state of excruciating insanity. You smoked your last cigarette 
yesterday. Concentrate. Try to understand what is happening to you."

He kept taking the Drome cigarette apart and munching bits of it. » (Lolita 297)

15. Library of Congress, Washington D.C., Manuscript Division. Vladimir Vladimirovich Nabokov Papers, Lolita. A Screenplay. Draft in English, on index cards, n. d., container \#16, folder \#2, index card \#28.

16. De manière assez ironique, une série de photographies de Nabokov et son épouse dans leur quotidien à Montreux paraîtra dans Life en 1964.

17. Comme il le note dans la nouvelle "Lance", Nabokov a bien conscience de l'impact des images de la publicité sur l'imagination : "I am somewhat disappointed that I cannot make out her features. All I manage to glimpse is an effect of melting light on one side of her misty hair, and in this, I suspect, I am insidiously influenced by the standard artistry of modern photography and I feel how much easier writing must have been in former days when one's imagination was not hemmed in by innumerable visual aids, and a frontiersman looking at his first giant cactus or his first high snows was not necessarily reminded of a tire company's pictorial advertisement " (Stories, 635).

18. «But a different lot and another fate await the writer who has dared to evoke all such things that are constantly before one's eyes but which idle eyes do not see-the shocking morass of trifles that has tied up our lives, and the essence of cold, crumbling, humdrum characters with whom our earthly way, now bitter, now dull, fairly swarms; has dared to make them prominently and brightly visible to the eyes of all men by means of the vigorous strength of his pitiless chisel. Not for him will be the applause, no grateful tears will he see, no souls will he excite with unanimous admiration; (...) for the judgment of his time does not admit that a man requires a good deal of spiritual depth in order to be able to throw light upon an image supplied by base life and to turn it into an exquisite masterpiece (...) » (Nicolai Gogol 105-106).

19. Avec Lolita, Nabokov met au point le système d'écriture qui sera le sien jusqu'à sa dernière œuvre inachevée, à savoir la rédaction de ses romans sur des petits bristols dont il n'utilise en général que le recto, et qu'il numérote et réorganise à sa guise. Cette technique lui permet de commencer la rédaction où il le souhaite, et d'ajouter au fil de l'écriture des bristols pour mettre en place le système complexe de motifs qui sont entrelacés dans ses œuvres.

20. Library of Congress, Washington D.C., Manuscript Division. Vladimir Vladimirovich Nabokov Papers, Lolita, Index cards (unnumbered). Notes in English (1950-1955), container 11, folder 3 of 6. 21. Rappelons que Humbert envisage de se servir de l'utérus de Lolita comme source de production de nymphettes ("a litter of lolitas» Lolita 300), ce qui lui permettra de toujours assouvir son désir, une fois passé le court intervalle, entre neuf et quatorze ans, où les nymphettes peuvent éclore, même si cela implique de recourir à l'inceste entre père et fille puis entre grand-père et petite-fille (Lolita 174), qu'il présente comme la solution idéale.

22. Library of Congress, Washington D.C., Manuscript Division. Vladimir Vladimirovich Nabokov Papers, Lolita, Index cards (unnumbered). Notes in English (1950-1955), container 11, folder 3 of 6. 23. Samuel Schuman voit dans "Tristram" un clin d'œil à Sterne qui souligne le caractère satirique de cet extrait via la référence intertextuelle (Schuman 2), mais la référence à l'amour, grimée dans le titre bien réel du magazine d'où Nabokov a tiré un certain nombre des phrases et expressions qui suivent la référence à Tristan, pourrait aussi être une allusion au récit courtois de l'histoire de Tristan et Yseult, histoire d'amour tragique et réciproque qui sert de contrepoint à celle de Humbert et Lolita, et à celles qui sont narrées dans ce type de magazine, que ce soit les aventures sentimentales en bande dessinée de Patsy Walker ou les tumultueuses amours de Marylin Monroe et Joe di Maggio (« the Joe-Roe marital enigma making yaps flaps »).

24. Voir note d'Alfred Appel illustrée par un dessin de Nabokov (Lolita 430-431).

25. Michael Wood a été l'un des premiers à considérer Humbert « less of a snob than many of his scholarly readers, who have seen in Lolita a condemnation of America's shallow, mass-managed culture » (Wood 115). En outre, au fil des entretiens, Nabokov ne cesse de rappeler son affection 
et son attachement à la fois pour les États-Unis et pour Lolita. Une récente biographie qui s'attache uniquement à la vie américaine de Nabokov est parue et se donne pour objet de démontrer l'attachement de Nabokov pour son pays d'adoption (Robert Roper. Nabokov in America, On the Road to Lolita. New York : Bloomsbury, 2015).

26. "Dolores styles herself with the detail and generality of mass culture [...] Her New World ideals fix only on a synchrony of movie stars, jukeboxes, and the right sneakers, sandals, and loafers [...] Dolores's America, like little Dolores herself, nestles in assorted clichés » (Rothstein 28).

27. Stringer-Hye voit ce trait comme également ce qui séduit le lecteur : « Many cultured readers condemn, along with Humbert, Lolita's shallowness, her taste for fudge, pop music and gooey sundaes. Yet it is the eerie vulgarity of her charms that seduces Humbert first, and the reader next, into her enchanted sphere ». (Stringer-Hye 154)

28. «I think I was born a painter-really!-and up to my fourteenth year, perhaps, I used to spend most of the day drawing and painting and I was supposed to become a painter in due time. But I don't think I had any real talent there " (Strong Opinions 17).

29. Ralph Waldo Emerson, "An Oration delivered before the Phi Beta Kappa Society, at Cambridge, August 31, 1837 ", in Nature; Addresses and Lectures (1849).

\section{RÉSUMÉS}

Cette étude s'attache à interroger les dispositifs intertextuels de Lolita en revenant non pas, comme d'ordinaire, sur l'interdiscursivité proprement littéraire ou artistique, mais sur la manière dont sont intégrés des textes et des images qui relèvent de la culture de masse des ÉtatsUnis après 1945. Grâce aux notes préparatoires au roman conservées à la Bibliothèque du Congrès, cette étude propose de comparer la manière dont Nabokov combine le matériau nonlittéraire à son écriture avec sa pratique intertextuelle plus traditionnelle, afin d'identifier la place et le rôle de ces références dans sa poétique.

This paper focuses upon intertextuality in Nabokov's Lolita, but not through literary allusion. Instead, this study analyzes the way the author integrates non-literary material taken from postWorld War 2 American mass culture into the textual fabric. Thanks to the preparatory notes to the novel kept at the Manuscript Division of the Library of Congress, one can compare the way Nabokov weaves the non-literary elements into the fabric of his writing with his more traditional intertextual practice, so as to define the role of popular culture in his aesthetics.

\section{INDEX}

Keywords : Nabokov, Lolita, mass culture, popular culture, magazine, advertizing, ad, intertextuality, intermediality, genetic criticism

Mots-clés : Nabokov, Lolita, culture de masse, culture populaire, magazine, publicité, intertextualité, intermédialité, critique génétique 


\section{AUTEURS}

\section{MARIE BOUCHET}

Maître de Conférences

Université de Toulouse

marie.bouchet@univ-tlse2.fr 